\title{
Importance of Clinical Assessment in Selecting Patients for Pulmonary Arteriography*
}

PAUL D. STEIN, MD, FACC ${ }^{\dagger}$

PARK W. WILLIS, III, ML, FACC $\ddagger$

JAMES E. DALEN, MD, FACC $\$$

Detroit and Ann Arbor, Michigan

Worcester, Massachusetts:

A recent report ${ }^{1}$ indicates an exceedingly high incidence rate ( 73 percent) of negative pulmonary arteriograms in patients with suspected pulmonary embolism. All of these patients had a "suitable clinical history," hypoxemia and positive perfusion scans. In view of this apparent failure of clinical evaluation and noninvasive tests, it was concluded that overdiagnosis and overtreatment can be avoided only with wide use of pulmonary arteriography. The clear implication was that pulmonary embolism can be neither identified with reasonable certainty nor excluded in a vast majority of suspected instances unless pulmonary arteriograms are performed. It was suggested that "the emperor has no clothes." We strongly disagree. We believe that the clinical manifestations of pulmonary embolism are now sufficiently recognizable to permit a correct presumptive diagnosis or a correct exclusion in a large majority of patients. We believe that an incidence rate of 70 to 80 percent of negative pulmonary arteriograms in patients with suspected pulmonary embolism reflects a serious underevaluation of the important clinical manifestations of pulmonary embolism. Although the signs and symptoms of pulmonary embolism are nonspecific, the manifestations, when considered as a group, form a set of syndromes that are strongly suggestive. Conversely, the absence of key clinical features is strong evidence for exclusion. The purpose of this communication is to suggest that the clinical manifestations, when carefully and fully evaluated, are sufficiently

From the Departments of Medicine and Surgery, Henry Ford Hospital, Detroit, Michigan ${ }^{\dagger}$; the Departrnent of Medicine, University of Michigan, College of Medicine, Ann Arbor, Michigan $\ddagger$ and the Department of Medicine, University of Massachusetts, Worcester, Massachusetts. $\S$

Address for reprints: Paul D. Stein, Henry Ford Hospital, 2799 West Grand Boulevard, Detroit, Michigan 48202. typical of acute pulmonary embolism to permit a judicious selection of patients for pulmonary arteriography and thus reduce the reported incidence of negative pulmonary arteriograms.

\section{Evaluation of Clinical Manifestations}

The most typical diagnostic feature of acute pulmonary embolism is the sudden and unexplained onset of severe dyspnea. This single historical point is highly suggestive of acute pulmonary embolism. The absence of this symptom is a strong argument against the diagnosis. Hemoptysis and friction rub, which are manifestations of pulmonary infarction, are absent in more than 70 percent of patients with acute pulmonary embolism $^{2}$ and are more often absent with massive than with submassive embolism. ${ }^{2}$

Deep venous thrombosis is a highly suggestive predisposing factor because acute pulmonary embolism is associated with thrombi of the lower limbs in the vast majority of patients. Nevertheless, an absence of symptoms or signs of deep venous thrombosis by no means excludes the diagnosis because clinical manifestations of deep venous thrombosis are absent in more than half of patients with massive acute pulmonary embolism. ${ }^{2}$ However, factors that predispose to deep venous thrombosis are almost invariably present. Prolonged immobility, bed rest, congestive heart failure, a recent operative procedure, chronic debilitating disease, fracture of the leg or pelvis, pregnancy, long automobile rides and oral contraceptive agents are predisposing factors that are expected among the constellation of factors necessary for a clinical diagnosis. ${ }^{3}$ The absence of predisposing factors is unusual, and when this is the case, diagnosis of pulmonary embolism is in question.

\footnotetext{
- Editorials published by the Journal reflect the views of the authors and do not necessarily represent the views of the Journal or of the American College of Cardiology.
} 
The respiratory rate in acute pulmonary embolism is usually increased (greater than $16 / \mathrm{min}$ ). ${ }^{2,4}$ A normal respiratory rate decreases the likelihood that major pulmonary embolism has occurred. The signs of acute cor pulmonale, if present, are supportive of the diagnosis. These may include a prominent $A$ wave visible in the neck veins, a right ventricular impulse, a right ventricular third or fourth sound and an accentuated pulmonary component of the second heart sound. A friction rub, if present, suggests the complication of pulmonary infarction.

The chest roentgenogram is strongly suggestive of pulmonary embolism if it is normal in a severely dyspneic patient. A dome of the diaphragm may he elevated. A localized radiodensity may represent pulmonary infarction, and a small effusion may accompany the infarction. Large pleural effusions are rare in pulmonary embolism and suggest another disease process.

The electrocardiogram may be strongly supportive of the clinical diagnosis of acute pulmonary embolism. The most frequent abnormality is a nonspecific change of the $\mathrm{S}$ - $\mathrm{T}$ segment or $\mathrm{T}$ wave that occurs in more than 40 percent of patients with massive or submassive acute pulmonary embolism..$^{5}$ Even minor changes of the S-T segment or $\mathrm{T}$ wave are supportive of the diagnosis. A normal electrocardiogram is uncommon in acute pulmonary embolism and is found in only 6 percent of patients with massive pulmonary embolism and 23 percent with submassive embolism. ${ }^{5}$ Complete right bundle branch block, right axis deviation, an $\mathrm{S}_{1} \mathrm{Q}_{3} \mathrm{~T}_{3}$ pattern or $\mathbf{P}$ pulmonale occurs in approximately one third of patients with massive or submassive pulmonary embolism. ${ }^{5}$ Left axis deviation is found as frequently as right axis deviation in acute pulmonary embolism, and therefore does not exclude the diagnosis. ${ }^{5}$

The partial pressure of oxygen in arterial blood, if it is low, is evidence in favor of pulmonary embolism. ${ }^{6}$ It is not generally recognized, however, that the partial pressure of oxygen in the arterial blood of elderly subjects is often low. Normal arterial oxygen levels may occur in patients with submassive pulmonary embolism and rarely in patients with massive embolism.

A negative nuclear lung scan excludes the diagnosis of clinically important pulmonary embolism. In a patient free of cardiac or pulmonary disease, an abnormal perfusion lung scan is strongly suggestive of pulmonary embolism. However, many disorders can cause abnormalities of pulmonary perfusion. These include chronic obstructive pulmonary disease, pneumonia, asthma, atelectasis, pulmonary congestion, mitral stenosis (even in the absence of chest X-ray evidence of pulmonary congestion), lung resection, carcinoma of the lung; pulmonary arteriovenous fistula, pleural effusion, and no doubt others. A perfusion scan that shows multiple segmental defects in regions that are normal in a ventilation scan greatly increases the probability of pulmonary embolism. ${ }^{7}$

Faithful and understanding evaluation of the signs, symptoms, predisposing factors and plain chest roentgenograms, in combination with the prudent use of ar- terial oxygen levels and lung scans, permits one to establish a diagnosis of pulmonary embolism with reasonable assurance in many patients. Conversely, the absence of important clinical manifestations, particularly sudden unexplained dyspnea, makes the diagnosis of acute pulmonary embolism unlikely. What, then, is the role of pulmonary arteriography?

\section{Role of Pulmonary Arteriography}

Indications: Pulmonary arteriography is useful to verify the diagnosis of pulmonary embolism in patients who have clear clinical manifestations of the syndrome. The risks of treatment with thrombolytic agents, inferior vena caval interruption and pulmonary embolectomy are sufficient to require that the diagnosis be firmly established before the initiation of therapy; and arteriography is the most definitive diagnostic procedure. In patients with clear clinical manifestations and an abnormal lung scan in the absence of cardiac or pulmonary disease, it is reasonable to treat with anticoagulant agents without performing pulmonary arteriograms. If invasive or thrombolytic therapy is contemplated, then pulmonary arteriography is mandatory. If the diagnosis is questionable after careful evaluation of the clinical manifestations, then arteriography is indicated. On the other hand, if the clinical manifestations do not suggest pulmonary embolism, as, for example, the gradual exacerbation of dyspnea in a patient with chronic obstructive pulmonary disease, then pulmonary arteriography is not indicated.

Errors in interpretation: Even though pulmonary arteriography is the most definitive diagnostic procedure, it too is subject to errors of interpretation that relate to technique as well as to the time of occurrence of embolism relative to the arteriogram. Thromboemboli undergo lysis and fragmentation, but the time required for resolution is not clearly established. In dogs with pulmonary embolism induced with freshly formed clots, considerable lysis and fragmentation occurred within 24 hours. $^{8}$ In patients, noticeable resolution has been observed within 24 hours and complete resolution of massive thromboemboli has been observed within 2 weeks. ${ }^{9}$ Therefore, pulmonary arteriograms obtained within days after the onset of symptoms would be of the most assistance in reaching a correct diagnosis. When performed after a long delay, the pulmonary arteriogram is likely not to be diagnostic.

Demonstration of small pulmonary emboli: Thromboemboli that are too small to occlude major branches of the pulmonary artery frequently cannot be visualized clearly after an injection in the main pulmonary artery. Selective injections in the right or left pulmonary artery are necessary in this situation. If visualization remains unclear, injection in distal segments, sometimes using cineangiography rather than cut films, may be helpful. ${ }^{10}$ To demonstrate very small emboli, wedge arteriograms may be useful. With this technique, pulmonary emboli in vessels less than $2 \mathrm{~mm}$ in diameter have been demonstrated. ${ }^{11}$ Thus, the yield from pulmonary arteriography relates to the persistence of the arteriographer, the selectivity of injections and the 
quality of the filming 1,echnique. For satisfactory pulmonary arteriography, the patient must be able to remain immobile and withstand the discomfort of a sufficient number of injections to allow complete definition of the arteriographic features. In patients with massive pulmonary embolism, the diagnosis can be established from arteriograms obtained during the first injection in the main pulmonary artery. In contradistinction, in patients with symptomatic pulmonary disorders of other etiology, in whorn an effort is being made to exclude assaciated but unlikely pulmonary embolism, multiple selective and superselective injections may be required.

Risks: The risk of pulmonary arteriography is low (2 to 4 percent morbidity)..$^{4,12}$ Nevertheless, pulmonary arteriography, in our judgment, should not be used to "rule out" pulmonary embolism when clinical assessment may suffice. A physician must not feel obligated to perform a pulmonary arteriogram to exclude pulmonary embolism that is improbable on the basis of the clinical presentation. 'The yield of useful information under such circumstances is low and the complexity and associated discomfort of an extended procedure searching for minute emboli lead to an increased and unjustified risk of morbidity from the procedure.
The presence of a localized and otherwise unexplained perfusion defect in the lung scan of a patient with prominent pulmonary disease does not in our judgment invalidate the clinical evaluation of the patient. Numerous injections of contrast material would be required to arrive at a negative arteriographic diagnosis in such patients. Nonspecific arteriographic abnormalities, such as an absence of fine branching, and regional hypoperfusion are of no value in the presence of preexisting pulmonary disease. ${ }^{13}$ An important practical issue is that the patient, ill from another active discase process, often cannot be sufficicntly caoperative to undergo the numerous injections necessary for an adequate examination.

\section{Conclusions}

In our judgment, pulmonary arteriography should not be used to exclude pulmonary embolism when the clinical manifestations can reasonably exclude the diagnosis. We suggest that there are clinical manifestations of pulmonary embolism upon which one may rely with enough assurance to permit a judicious selection of patients for pulmonary arteriography and thereby reduce the prevalence of negative pulmonary arteriograms with their attendant morbidity.

\section{References}

1. Robin ED: Overdiagnosis and overtreatment of pulmonary embolism: the emperor may have no clothes. Ann Intern Med 87: 775-781, 1977

2. Wenger KN, Stein PD, Willis PW III: Massive acute pulmonary embolism. The deceivingly nonspecific manifestations. JAMA 220:843-844, 1972

3. Willis PW III, Coon WW: Pulmonary embolism and infarction. In, Cardiac Emergencies (Mason DT, ed). Baltimore, Williams \& Wilkins, 1978, p 224-248

4. Dalen JE: Dlagnosis of acute pulmonary embolism. In, Puimonary Embolism (Dalen JE, ed). New York, Medcom Press, 1972, p 28-39

5. Stein PD, Dalen JE, Mcilntyre KM, Sasahara AA, Wenger NK, Willis PW III: The electrocardiogram in acute pulmonary embolism. Prog Cardiovasc Dis 17:247-257, 1975

6. Szucs MM, Brooks HL, Grossman W, Banas JS, Meister SG, Dexter L, Dalen JE: Diagnostic sensitivity of laboratory findings in acute pulmonary embolism. Ann Intern Med 74:161-166, 1971

7. McNeil BJ: A diagnostic strategy using ventilation-perfusion studies in patients suspect for pulmonary embolism. J Nucl Med 17: 613-616, 1976

8. Mathur VS, Dalen JE, Evans H, Haynes FW, Pur-Shahriari AA, Stein PD, Dexter L: Pulmonary angiography one to seven days after experimental pulmonary embolism. Invest Radiol 2:304-312, 1967

9. Dalen JE, Banas JS, Brooks HL, Evans GL, Paraskos JA, Dextęr L: Resolution rate of acute pulmonary embolism in man. I Engl J Med 280:1194-1198, 1969

10. Meister SG, Brooks HL, Szucs MM, Banas JS Jr., Dexter L, Dạlen JE: Pulmonary cineangiography in acute pulmonary embolism. Am Heart J 84:33-37, 1972

11. Stein PD: Wedge arteriography for the identification of pulmonary emboli in small vessels. Am Heart J 82:618-623, 1971

12. National Cooperative Study. Pulmonary angiography. In, The urokinase pulmonary embolism trial. Circulation 47:Suppl II:II 39-1I 45,1973

13. Stein PD, Leu JD, Welch MH, Guenter CA: Pathophysiology of the pulmonary circulation in emphysema associated with alpha antitrypsin deficiency. Circulation 43:227-239, 1971 